\title{
Development and Validation of an Interleukin-6 Nomogram to Predict Primary Non-response to Infliximab in Crohn's Disease Patients: From Bedside to Bioinformatics.
}

\author{
Yueying Chen ${ }^{1}$, Hanyang $\mathrm{Li}^{1}$, Qi Feng ${ }^{1}$, and Jun Shen ${ }^{1}$ \\ ${ }^{1}$ Shanghai Jiao Tong University
}

December 1, 2020

\begin{abstract}
Background: The primary nonresponse (PNR) rate of Infliximab (IFX) varies from $20 \%$ to $46 \%$ for the treatment of Crohn's disease $(\mathrm{CD})$. Detected PNR reduces the improper use of specific treatments. To date, there is hardly any knowledge regarding early markers of PNR. The aim of this study was to evaluate the role of Interleukin-6 (IL-6) as an early predictor of PNR of IFX for the treatment of CD. Methods: We enrolled 322 bio-naïve patients diagnosed with CD from January 2016 to May 2020. Primary response was determined at week 14. Multivariable logistic regression was used to construct prediction models. The discrimination, calibration and clinical validity of the models in the validation cohort were assessed by area under the curve (AUC), calibration and decision curves analyses. GEO data were analyzed to identify potential mechanisms of IL-6 in IFX therapy for CD. Results: PNR occurred in 31.06\% (100 of 322) patients who were assessable at week 14. IL-6 levels significantly decreased after IFX therapy $(\mathrm{P}<0.001)$. The validation model containing IL-6 presented enhanced discrimination with an AUC of 0.908 and high calibration. Decision curve analysis (DCA) indicated that the model added extra predictive value. GEO data confirmed the IL-6 levels were increased in the PNR group and IL-6-related differently expressed genes (DEGs) were enriched in the inflammatory response. Conclusion: We concluded that IL-6 may be used as a predictive factor to assess the risk of PNR to IFX therapy.
\end{abstract}

\section{Introduction}

Crohn's disease $(\mathrm{CD})$ is a chronic inflammatory disease with a relapsing history. The incidence and prevalence of $\mathrm{CD}$ have both increased worldwide and this disease has gradually become a more severe socioeconomic burden (1). Recently, the treatment of CD entered a new era. Anti-tumor necrosis factor (anti-TNF) therapy has been the first-line therapy for treating CD according to ECCO guidelines (2). Infliximab (IFX) is the most widely used anti-TNF agent that promotes mucosal healing and changes the natural history of the disease (3). However, there are still $20-46 \%$ of CD patients that show primary non-response (PNR) to IFX (4, 5). Leapfrogging IFX in PNR patients to other medications such as anti-IL-12/23 monoclonal antibodies or anti-leucocyte adhesion molecules avoid ineffective processes of IFX and saves medical resources. Therefore, developing a model to predict PNR to IFX in CD patients will help doctors make better decisions for CD patients.

Previous studies have proposed several factors to predict the efficacy of IFX in CD, including age, BMI, previous surgery history (6), disease behavior (7), disease duration (8), and pro-inflammatory biomarkers (9). Despite evidence, a clinical prediction model is still lacking to assess the possibility of PNR prior to IFX administration based on clinical and biochemical markers. As a chronic inflammatory disease, the regulation of cytokines is associated with the pathogenesis and progression of CD (10). IL-6 is a proinflammatory 
factor that can exacerbate inflammation by promoting the survival of $\mathrm{T}$ cells and the secretion of other cytokines (11). $I L-6$ levels were shown to be elevated in CD patients compared to healthy individuals (12). A randomized trial showed that an anti-IL-6 antibody promoted a clinical response and clinical remission in CD patients (13). Furthermore, IL-6 served as a predictor of PNR to anti-TNF treatment in CD and patients failing anti-TNF therapy showed increased expression of IL-6 and persistent IL-6 pathway activity $(14,15)$. A prospective, multicenter study confirmed the predictive roles of IL-6 in patients treated with anti-TNF therapy (16). Additionally, the single nucleotide polymorphisms of IL-6 may be a promising tool for identifying CD patient response to IFX therapy (17).

To promote individualized treatment in CD patients, it is crucial to identify predictors to estimate PNR to IFX. According to previous work, IL-6 levels may alter after IFX treatment in CD patients. Meanwhile, different IL-6 levels present before treatment may contribute to the contrasting response to IFX therapy. Detecting loss of PNR reduces inaccurate treatments and a predictive model determining PNR to IFX in bio-naive CD is lacking. Therefore, we aimed to evaluate PNR of IFX therapy in bio-naive CD patients, to identify the predictive value of IL- 6 in IFX therapy and to construct a model predicting PNR to IFX therapy based on clinical information and IL-6 levels.

\section{Methods}

\section{Patients and samples}

A retrospective, single-center, observational study was performed for CD cases in the Division of Gastroenterology and Hepatology, Renji hospital from January 2016 to May 2020. Patients enrolled in this study from January 2016 to June 2019 and from July 2019 to May 2020 were assigned to the training and the validation cohorts, respectively. CD was diagnosed based on the ECCO consensus (18). Bio-naïve patients were included and induced with $5 \mathrm{mg} / \mathrm{kg}$ of IFX. Baseline characteristics were collected prior to treatment.

Levels of biochemical indicators, including IL-6, albumin, hemoglobin, platelets, erythrocyte sedimentation rate (ESR) and CRP were obtained from previous blood analysis, and blood samples were collected for each participant before and after IFX therapy. The Westergren method and nephelometry were used to measure CRP and ESR levels. Enzyme Linked Immunosorbent Assay (ELISA) was used to detect IL-6 levels using a DPC IMMULITE 1000 system of Siemens, and the average CV value about 8.33\%. The Clinical Laboratory Department (Renji Hospital, School of Medicine, Shanghai Jiao Tong University, China) performed an analysis of each sample in duplicate. This study was approved by the IRB of Shanghai Jiaotong University School of Medicine, Renji Hospital Ethics Committee (KY2020-115). The informed consent of this study was waived by ethics committee, because it was retrospective and the noninvasive to patients. The privacy of patients was protected, and the results were used for comparative study only.

\section{Outcomes and definitions}

Our study defined the primary outcome as the proportion of response to IFX, which was determined when achieving clinical response or remission at week 14 (after three IFX injections and before the $4^{\text {th }}$ ) using an MDT (multi-disciplinary team) of experienced experts combined with endoscopic and radiological examinations $(19,20)$. PNR was defined as: (1) failure to achieve clinical response or clinical remission, clinical response and clinical remission have been defined as a decrease in Crohn's Disease Activity Index (CDAI) [?] 100 or total CDAI [?] 150, and CDAI [?] 150, respectively $(2,21)$. (2) need for treatment modification (discontinuation, escalation or surgery) $(5,22,23)$. 


\section{Statistical analysis}

Results for continuous variables were represented as mean (SDs) or median (interquartile ranges [IQRs]). Categorical variables were shown as proportions. Univariate logistic regression was applied to analyze the relationships between different factors and PNR to IFX therapy. A Chi-square test was used to compare categorical variables. The $t$ test or Mann-Whitney $U$ test were used to compare continuous variables.

Multivariable regression models with forward stepwise likelihood ratio algorithms were used to develop models predicting the response to infliximab $5 \mathrm{mg} / \mathrm{kg}$ through 14 weeks. Akaike information criterion (AIC) was performed to assessed the goodness of fit of two models. Discrimination of the prediction models was evaluated by receiver operating characteristics (ROC) analysis and presented as area under the curve (AUC). Odds ratios (OR) having $95 \%$ confidence intervals (CI) of final predictors were calculated. Calibration curves were used to assess the calibration of nomograms by comparing the predicted and observed probabilities. The clinical effectiveness of the models was assessed using decision curve analysis (DCA). Integrated Discrimination Improvement (IDI) was a reclassification measures showed the difference in discrimination slopes of two models, and was used to assess the improvement of risk differences between cases and non-cases (24-26). All statistical analyses were performed using SPSS 25.0 and R 3.6.3 with a statistical significance of $P<$ 0.05 .

\section{Bioinformatic analysis}

\section{Data source}

A gene expression microarray dataset (GSE111761) of Schmitt's study from the GEO database was selected (27). GSE111761 contained 3 samples from anti-TNF non-responders and 3 from responders. The patients were diagnosed as CD and defined as responders or non-responders when they had ongoing anti-TNF therapy for over 3 months. The GSE111761 dataset was available on the GPL13497 platform (Agilent-026652 Whole Human Genome Microarray 4x44K v2).

\section{Identification of differentially expressed genes (DEGs)}

T R software (version 3.6.3) and the limma package in Bioconductor were used to detect DEGs in GSE111761 (28). DEGs were identified using selection criteria of an adjusted $\mathrm{P}$ value $<0.05$ and $|\log \mathrm{FC}|>1.0$.

\section{Enrichment analysis}

Gene Set Enrichment Analysis (GSEA) and Gene Ontology (GO) analyses were performed using the ClusterProfile package in Bioconductor with a statistical significance of $P<0.05$ (29).

\section{Protein-protein interaction (PPI) network construction}

PPI network reveals the specific and unspecific interactions of proteins, and promotes to identify therapeutic target (30,31). STRING (version 11.0), a freely accessible database that collects, scores and integrates data, was used to predict functional relationships between proteins (32). A PPI network of genes with a score $>$ 0.4 in STRING was constructed using Cytoscape software (version 3.7.2) (33). The degree of protein nodes was calculated using the Cytoscape plugin CytoHubba to identify hub genes (34). Hub genes were selected with a score [?] 4.5 based on the EPC algorithm.

\section{Construction of regulatory network}

The network of genes and their corresponding miRNAs and lncRNAs was constructed using StarBase, a publicly available database mainly focusing on miRNA-target interactions (35). Transcription factors (TFs) were downloaded from TRRUST (http://www.grnpedia.org/trrust/), a public database for predicting TFs of various genes through DNA sequences (36). These tools were combined to construct a multi-factor regulation network. 


\section{Results}

\section{Baseline characteristics and univariate analyses}

From a total of 322 active CD patients receiving $5 \mathrm{mg} / \mathrm{kg}$ IFX induction therapy, 223 and 99 were assigned to training and validation groups, respectively (Figure 1 ). Disease behavior was merged into two categories, including nonstricturing and nonpenetrating (B1) into one category, and stricturing and/or penetrating (B2/B3) subtypes into another category. The baseline characteristics are shown inTable 1 . The incidence of PNR in the training cohort was $30.0 \%(\mathrm{n}=67)$, while the incidence in the validation cohort was $33.3 \%$ $(\mathrm{n}=33)$. Baseline characteristics of patients were similar between the two cohorts. Univariate regressions showed that BMI $(\mathrm{P}<0.001)$, disease behavior $(\mathrm{P}<0.001)$, CRP levels $(\mathrm{P}=0.001)$, and IL-6 levels $(\mathrm{P}=$ 0.002) were strongly associated with PNR to IFX treatment (Table 2 ).

IL-6 levels were measured in 258 of the studied 322 patients before initiation and at week 14 before the $4^{\text {th }}$ IFX injection. IL-6 levels were found to significantly decrease after IFX therapy $(\mathrm{P}<0.001)$ and were obviously different between PNR and response groups $(\mathrm{P}=0.001)$ (Supplementary Figure 1 ).

\section{Development of prediction models and nomogram construction}

Prediction models were developed using multiple logistic regression. Results suggested BMI, disease behavior, CRP levels and IL-6 levels were determined as predicting factors. BMI, disease behavior and CRP have been considered as predictors for loss of response. To identify the predictive effects of IL- 6 and whether it increased predictive value by developing a logical model, we built two predictive models. One model contained IL-6 and the other did not. The diagnostic equation of model 1 was: $\operatorname{logitP}=1.162-0.217^{*} \mathrm{BMI}+1.281 *$ behavior $+0.025 *$ CRP. For model 2, the diagnostic equation was: $\operatorname{logitP}=0.144-0.212^{*} \mathrm{BMI}+1.252 *$ behavior $+0.024 *$ CRP $+0.237^{*}$ IL- 6 . The multivariate regression analyses of the two models are shown in Table 3 . These two models containing these independent predictors are presented as nomograms (Figure 2 ). According to the results of multiple logistic regressions, nomograms containing these independent predictors were developed to calculate the risk of PNR based on the total points linked to these risk factors (BMI, behavior, CRP, and IL-6) (Figure 2 ).

\section{Validation of prediction models}

Efficacies of the two models were compared to the validation cohort. AIC was 111.3 and 99.1 of model1 and model2, respectively. ROC curves of model 1 and model 2 are presented in Figure 3 . ROC analysis indicated an AUC of 0.813 (95\% CI: 0.729 to 0.897) in model 1 and 0.908 (95\% CI: 0.851 to 0.966) in model 2. The $\mathrm{P}$ value $=0.005$ of DeLong's test showed that an AUC of model 2 was significantly better

than model 1. Calibration plots showed that the average differences (E aver) were $2.5 \%$ and $1.8 \%$, and the maximal differences (E max) were $7.0 \%$ and $4.4 \%$ between the predicted and the calibrated probabilities in model 1 and model 2, respectively. Meanwhile, there were no significant differences between the predicted and calibrated probabilities $\left(P_{1}=0.844, P_{2}=0.947\right)$, which showed the calibration of two models were well established. The clinical utility of these two models was presented by the DCA. Results showed that if the risk thresholds were between $12 \%$ and $85 \%$, model 2 predictive nomograms could be used to predict PNR rate and adds additional net benefit when compared to model 1. Furthermore, nomogram 2 cooperated with IL-6 levels and demonstrated better clinical practicality compared with nomogram 1. To explore additional benefits of IL-6 levels in the prediction of response to IFX, we compared IDI based on the validation cohort. IDI analysis suggested that adding IL-6 significantly improved this improvement index (IDI $=19 \%$; $95 \%$ CI, $0.10-0.28 ; \mathrm{P}<0.000)$.

\section{Identification of pathway and regulatory network of IL-6}

To identify potential pathways and regulatory network of IL-6 in IFX therapy of CD, the GSE111761 dataset with samples from anti-TNF non-responders and responders were included to identify DEGs. The GSE111761 dataset contained gene expression profiles of isolated lamina propria mononuclear cells derived 
from three responders and three non-responders to anti-TNF therapy from six CD patients. The samples were obtained from the patients with anti-TNF therapy for over 3 months and the Simple Endoscopic Score for Crohn's Disease (SES-CD) $<5$. We used the limma package to identify DEGs in the GSE111761 with a $\mathrm{P}<0.05$ and $|\log \mathrm{FC}|>1$. There were 2228 DEGs including 1384 upregulated genes and 844 downregulated genes and IL-6 expression levels were significantly elevated in PNR patients (Supplementary Figure 2 ).

Enrichment analyses of IL-6 related DEGs

We investigated the connections between $I L-6$ and other DEGs. Genes with a $\mathrm{P}<0.05$ were selected for GSEA analysis. Results indicated that $I L-6$ and its relative genes were mainly involved in pathways associated with inflammation and immunity. Inflammatory response showed the highest normalized enrichment score (NES) and it was enriched in the nonresponder group compared with responder group (Figure 4 and Supplementary Table 3). These results suggested that $I L-6$ and its related genes played a role in PNR to IFX by regulating the inflammatory response.

PPI network construction of IL-6 related genes determined hub genes

Although GSEA analysis identified the inflammatory response as the downstream regulator, specific potential $I L-6$ related genes were still unknown. Thus, PPI network analysis was used to understand DEGs in responders and non-responders to anti-TNF therapy. We selected $I L-6$ related genes with the top 50 highest correlation coefficients to create PPIs (Figure 5 ). There were 13 genes directly connected to $I L-6$. We analyzed these 13 genes and $I L-6$ using GO analysis. GO analysis indicated that $I L-6$ and its related genes were mainly enriched in the inflammatory response (Supplementary Figure 1 ). This result was consistent with our GSEA results. With a criteria of score [?] 4.5 based on the EPC algorithm using CytoHubba plugin, we identified five hub genes in PPI, including IL-6, IGF2, C5AR1, IFNLR1 and OSM .

Multi-factor regulation network construction

We found that the expression of Oncostatin M (OSM ) coordinated with $I L-6$ and also belonged to the inflammatory response in both GSEA and GO analysis. To identify potential biological mechanisms underlying the association between $I L-6$ and $O S M$, we used StarBase and TRRUST databases to predict miRNAs, lncRNAs, and TFs of $I L-6$ and $O S M$. We found a total of 10 miRNAs, $25 \operatorname{lncRNAs}$, and 1 TF shared in common with both $I L-6$ and $O S M$. Data of these two genes and their miRNAs, lncRNAs and TF were integrated into a regulatory network and visualized using Cytoscape software (Figure 6 ). LncRNAs were important factors in the expression of $I L-6$ and $O S M$ levels by bonding with miRNAs, while the transcriptional regulation of lncRNAs and miRNAs could be regulated by STAT3, a common TF of $I L-6$ and OSM . It was suggested that $I L-6$ and $O S M$ may be involved in the inflammatory response through a regulation network based on common lncRNAs, miRNAs and TF.

\section{Discussion}

Even though anti-TNF agents have shown to be effective in inducing clinical response and mucosal healing, there are still a considerable proportion of patients who do not respond (21). Previous studies have identified several predictors of PNR to IFX in CD, such as BMI, fecal calprotectin (37), proinflammatory biomarkers (9), and genetic markers (38). These studies have also established prediction models based on these factors. IL-6 is a proinflammatory cytokine and expression levels alter after IFX therapy. It is considered a good predictor of IFX response $(17,39)$. However, there are few prediction models for PNR to IFX treatment in CD. In this study, we showed that IL-6 levels before IFX therapy predicted the response to IFX treatment and alter after IFX therapy.

This retrospective study included $322 \mathrm{CD}$ patients who were naive to anti-TNF therapy and both their clinical and serological data were collected. Logarithmic transformation was performed for IL-6 levels to decrease the undulation of data. Consistent with previous reports, IL-6 levels were significantly reduced after 14 weeks of IFX therapy. Univariate and multivariate regression analyses found that BMI, disease behavior, CRP 
and IL-6 levels before IFX therapy independently predicted the response to IFX treatment. To explore the predictive ability of IL-6, we established two prediction models, one containing BMI, disease behavior and CRP levels, and the other including BMI, disease behavior, CRP levels, and IL-6 levels. Results of AIC and ROC in the validation cohort, indicated that the goodness of fit and discrimination of model containing IL-6 was better than the model not including IL-6. The calibration curve and DCA curve demonstrated a greater consistency and clinical validity in the model containing IL-6. IDI analysis showed that incorporation of IL-6 with BMI, behavior, and CRP significantly improved the discriminatory accuracy for PNR with IDI of $19 \%(\mathrm{P}<0.000)$. Furthermore, the improvement of IDI for discriminating PNR to IFX therapy is consistent with previous studies, such as Leal et. al (14), which revealed the predictive effect of IL-6 on risk of PNR to anti-TNF therapy in CD patients. Therefore, we suggested that IL-6 played a central role in assessing the possibility of PNR to IFX therapy.

Furthermore, we analyzed GEO data to verify the predictive power of IL-6. It was found that expression of $I L-6$ significantly increased in non-responders compared with responders to IFX therapy. GSEA and GO analyses of $I L-6$ and its relative genes with high correlation coefficients showed that these genes mainly were enriched in the inflammatory response. Through the construction of PPI networks, we found that $O S M$ directly connected to $I L-6$ and its expression was consistent with $I L-6$ in the inflammatory response. OSM regulates the production of proinflammatory cytokines such as IL- 6 through the JAK-STAT pathway (40). Studies demonstrated that OSM induced intestinal inflammation, while mechanisms remained unclear (41). Furthermore, $O S M$ has been considered a novel biomarker to predict the efficacy of anti-TNF therapy. One study showed that $O S M$ was enriched in CD mucosa and complete mucosal healing was more likely to occur in patients with low $O S M$ expression levels. Notably, the expression of $O S M$ was strongly correlated with PNR to IFX (42). Another clinical study suggested that $O S M$ was an appreciable biomarker in predicting the possibility of mucosal healing compared with fecal calprotectin, which indicated $O S M$ may be a predictive indicator for IFX therapy (43). To explore the common mechanisms of $I L-6$ and $O S M$ in IFX treatment, we found that they both shared 10 miRNAs, 25 lncRNAs and $1 \mathrm{TF}$, which provides potential targets and pathways research diving into deeper mechanistic studies.

Although this study developed a new prediction model based on clinical and serological data, it faced several limitations. First, as a retrospective study, we defined PNR through chart review rather than prospectively collected disease activity indices. To ensure homogeneousness of the study, all CD patients were evaluated by the same physician group over the duration of IFX treatment. Second, IL-6 levels were detected in peripheral blood rather than mucosal tissue. As a result, findings represented the state of the peripheral immune system instead of the inflamed mucosa. Furthermore, external validation is required to confirm the validity of these results in clinical practice.

\section{Conclusion}

This study found IL-6 levels altered after IFX therapy and added IL-6 to enhance the predictive value of PNR to IFX therapy in CD bio-naive patients. A novel prediction model was developed, including IL-6 levels combined with BMI, disease behavior and CRP levels. With this model, clinicians can estimate the risk of PNR to IFX therapy and select the optimal treatment for individual patients. Furthermore, this study also constructed a multi-factor regulation network of $I L-6$ and its relative gene $O S M$, providing stronger direction for exploring the predictive and therapeutic targets of IFX treatment in CD. Further investigation is needed to determine the association between IL-6 from either peripheral or inflamed mucosal and anti-TNF treatment responses. 


\section{Contributors}

Yueying Chen and Hanyang Li, collected the papers and analyzed data, analyzed the conclusions, drafted the manuscript; Qi Feng reviewed the data and conclusions; Jun Shen presented the idea of this paper, supported the funding, analyzed the conclusions, drafted and revised the manuscript.

\section{Acknowledgments}

Supported by grants from National Natural Science Foundation of China (No. 81770545, and 81701746) and MDT Project of Clinical Research Innovation Foundation, Renji Hospital, School of Medicine, Shanghai Jiaotong University (PYI-17-003).

\section{Declaration of Interests}

The authors declare that they have no known competing financial interests or personal relationships that could have appeared to influence the work reported in this paper.

\section{References}

1. Ng SC, Tang W, Ching JY, Wong M, Chow CM, Hui AJ, et al. Incidence and phenotype of inflammatory bowel disease based on results from the Asia-pacific Crohn's and colitis epidemiology study. Gastroenterology. 2013;145(1):158-65.e2.

2. Torres J, Bonovas S, Doherty G, Kucharzik T, Gisbert JP, Raine T, et al. ECCO Guidelines on Therapeutics in Crohn's Disease: Medical Treatment. Journal of Crohn's \& colitis. 2020;14(1):4-22.

3. Singh S, Fumery M, Sandborn WJ, Murad MH. Systematic review and network meta-analysis: first- and second-line biologic therapies for moderate-severe Crohn's disease. Alimentary pharmacology \& therapeutics. 2018;48(4):394-409.

4. Yokoyama K, Yamazaki K, Katafuchi M, Ferchichi S. A Retrospective Claims Database Study on Drug Utilization in Japanese Patients with Crohn's Disease Treated with Adalimumab or Infliximab. Advances in therapy. 2016;33(11):1947-63.

5. Roda G, Jharap B, Neeraj N, Colombel JF. Loss of Response to Anti-TNFs: Definition, Epidemiology, and Management. Clinical and translational gastroenterology. 2016;7:e135.

6. Billiet T, Papamichael K, de Bruyn M, Verstockt B, Cleynen I, Princen F, et al. A Matrix-based Model Predicts Primary Response to Infliximab in Crohn's Disease. Journal of Crohn's \& colitis. 2015;9(12):1120-6.

7. Sprakes MB, Ford AC, Warren L, Greer D, Hamlin J. Efficacy, tolerability, and predictors of response to infliximab therapy for Crohn's disease: a large single centre experience. Journal of Crohn's \& colitis. 2012;6(2):143-53.

8. Matsuoka K, Hamada S, Shimizu M, Nanki K, Mizuno S, Kiyohara H, et al. Factors predicting the therapeutic response to infliximab during maintenance therapy in Japanese patients with Crohn's disease. PloS one. 2018;13(10):e0204632.

9. Billiet T, Cleynen I, Ballet V, Claes K, Princen F, Singh S, et al. Evolution of cytokines and inflammatory biomarkers during infliximab induction therapy and the impact of inflammatory burden on primary response in patients with Crohn's disease. Scandinavian journal of gastroenterology. 2017;52(10):1086-92. 
10. Chen ML, Sundrud MS. Cytokine Networks and T-Cell Subsets in Inflammatory Bowel Diseases. Inflamm Bowel Dis. 2016;22(5):1157-67.

11. Hunter CA, Jones SA. IL-6 as a keystone cytokine in health and disease. Nature immunology. $2015 ; 16(5): 448-57$.

12. Engel T, Ben-Horin S, Beer-Gabel M. Autonomic Dysfunction Correlates with Clinical and Inflammatory Activity in Patients with Crohn's Disease. Inflamm Bowel Dis. 2015;21(10):2320-6.

13. Danese S, Vermeire S, Hellstern P, Panaccione R, Rogler G, Fraser G, et al. Randomised trial and open-label extension study of an anti-interleukin-6 antibody in Crohn's disease (ANDANTE I and II). Gut. 2019;68(1):40-8.

14. Leal RF, Planell N, Kajekar R, Lozano JJ, Ordas I, Dotti I, et al. Identification of inflammatory mediators in patients with Crohn's disease unresponsive to anti-TNF $\alpha$ therapy. Gut. 2015;64(2):233-42.

15. Soendergaard C, Seidelin JB, Steenholdt C, Nielsen OH. Putative biomarkers of vedolizumab resistance and underlying inflammatory pathways involved in IBD. BMJ open gastroenterology. 2018;5(1):e000208.

16. Bertani L, Caviglia GP, Antonioli L, Pellicano R, Fagoonee S, Astegiano M, et al. Serum Interleukin-6 and -8 as Predictors of Response to Vedolizumab in Inflammatory Bowel Diseases. J Clin Med. 2020;9(5).

17. Salvador-Martín S, López-Cauce B, Nuñez O, Laserna-Mendieta EJ, García MI, Lobato E, et al. Genetic predictors of long-term response and trough levels of infliximab in crohn's disease. Pharmacological research. 2019;149:104478.

18. Maaser C, Sturm A, Vavricka SR, Kucharzik T, Fiorino G, Annese V, et al. ECCO-ESGAR Guideline for Diagnostic Assessment in IBD Part 1: Initial diagnosis, monitoring of known IBD, detection of complications. Journal of Crohn's \& colitis. 2019;13(2):144-64.

19. Hanauer SB, Feagan BG, Lichtenstein GR, Mayer LF, Schreiber S, Colombel JF, et al. Maintenance infliximab for Crohn's disease: the ACCENT I randomised trial. Lancet (London, England). 2002;359(9317):15419.

20. Wong U, Cross RK. Primary and secondary nonresponse to infliximab: mechanisms and countermeasures. Expert opinion on drug metabolism \& toxicology. 2017;13(10):1039-46.

21. Ding NS, Hart A, De Cruz P. Systematic review: predicting and optimising response to anti-TNF therapy in Crohn's disease - algorithm for practical management. Alimentary pharmacology \& therapeutics. 2016;43(1):30-51.

22. Bar-Yoseph H, Levhar N, Selinger L, Manor U, Yavzori M, Picard O, et al. Early drug and anti-infliximab antibody levels for prediction of primary nonresponse to infliximab therapy. Alimentary pharmacology \& therapeutics. 2018;47(2):212-8.

23. Beltrán B, Iborra M, Sáez-González E, Marqués-Miñana MR, Moret I, Cerrillo E, et al. Fecal Calprotectin Pretreatment and Induction Infliximab Levels for Prediction of Primary Nonresponse to Infliximab Therapy in Crohn's Disease. Digestive diseases (Basel, Switzerland). 2019;37(2):108-15.

24. Kerr KF, McClelland RL, Brown ER, Lumley T. Evaluating the incremental value of new biomarkers with integrated discrimination improvement. American journal of epidemiology. 2011;174(3):364-74.

25. Pencina MJ, D'Agostino RB, Sr., D'Agostino RB, Jr., Vasan RS. Evaluating the added predictive ability of a new marker: from area under the ROC curve to reclassification and beyond. Statistics in medicine. 2008;27(2):157-72; discussion 207-12.

26. Steyerberg EW, Vickers AJ, Cook NR, Gerds T, Gonen M, Obuchowski N, et al. Assessing the performance of prediction models: a framework for traditional and novel measures. Epidemiology (Cambridge, Mass). 2010;21(1):128-38. 
27. Schmitt H, Billmeier U, Dieterich W, Rath T, Sonnewald S, Reid S, et al. Expansion of IL-23 receptor bearing TNFR2+ T cells is associated with molecular resistance to anti-TNF therapy in Crohn's disease. Gut. 2019;68(5):814-28.

28. Ritchie ME, Phipson B, Wu D, Hu Y, Law CW, Shi W, et al. limma powers differential expression analyses for RNA-sequencing and microarray studies. Nucleic acids research. 2015;43(7):e47.

29. Yu G, Wang LG, Han Y, He QY. clusterProfiler: an R package for comparing biological themes among gene clusters. Omics : a journal of integrative biology. 2012;16(5):284-7.

30. Petta I, Lievens S, Libert C, Tavernier J, De Bosscher K. Modulation of Protein-Protein Interactions for the Development of Novel Therapeutics. Molecular therapy : the journal of the American Society of Gene Therapy. 2016;24(4):707-18.

31. Maurel M, Obacz J, Avril T, Ding YP, Papadodima O, Treton X, et al. Control of anterior GRadient 2 (AGR2) dimerization links endoplasmic reticulum proteostasis to inflammation. EMBO molecular medicine. $2019 ; 11(6)$.

32. Szklarczyk D, Gable AL, Lyon D, Junge A, Wyder S, Huerta-Cepas J, et al. STRING v11: protein-protein association networks with increased coverage, supporting functional discovery in genome-wide experimental datasets. Nucleic acids research. 2019;47(D1):D607-d13.

33. Smoot ME, Ono K, Ruscheinski J, Wang PL, Ideker T. Cytoscape 2.8: new features for data integration and network visualization. Bioinformatics (Oxford, England). 2011;27(3):431-2.

34. Chin CH, Chen SH, Wu HH, Ho CW, Ko MT, Lin CY. cytoHubba: identifying hub objects and subnetworks from complex interactome. BMC systems biology. 2014;8 Suppl 4:S11.

35. Li JH, Liu S, Zhou H, Qu LH, Yang JH. starBase v2.0: decoding miRNA-ceRNA, miRNA-ncRNA and protein-RNA interaction networks from large-scale CLIP-Seq data. Nucleic acids research. 2014;42(Database issue):D92-7.

36. Farre D, Roset R, Huerta M, Adsuara JE, Rosello L, Alba MM, et al. Identification of patterns in biological sequences at the ALGGEN server: PROMO and MALGEN. Nucleic acids research. 2003;31(13):3651-3.

37. Pavlidis P, Gulati S, Dubois P, Chung-Faye G, Sherwood R, Bjarnason I, et al. Early change in faecal calprotectin predicts primary non-response to anti-TNFalpha therapy in Crohn's disease. Scandinavian journal of gastroenterology. 2016;51(12):1447-52.

38. Barber GE, Yajnik V, Khalili H, Giallourakis C, Garber J, Xavier R, et al. Genetic Markers Predict Primary Non-Response and Durable Response To Anti-TNF Biologic Therapies in Crohn's Disease. The American journal of gastroenterology. 2016;111(12):1816-22.

39. Suzuki Y, Matsui T, Ito H, Ashida T, Nakamura S, Motoya S, et al. Circulating Interleukin 6 and Albumin, and Infliximab Levels Are Good Predictors of Recovering Efficacy After Dose Escalation Infliximab Therapy in Patients with Loss of Response to Treatment for Crohn's Disease: A Prospective Clinical Trial. Inflamm Bowel Dis. 2015;21(9):2114-22.

40. Hermanns HM. Oncostatin M and interleukin-31: Cytokines, receptors, signal transduction and physiology. Cytokine \& growth factor reviews. 2015;26(5):545-58.

41. Thomas H. IBD: Oncostatin M promotes inflammation in IBD. Nature reviews Gastroenterology \& hepatology. 2017;14(5):261.

42. West NR, Hegazy AN, Owens BMJ, Bullers SJ, Linggi B, Buonocore S, et al. Oncostatin M drives intestinal inflammation and predicts response to tumor necrosis factor-neutralizing therapy in patients with inflammatory bowel disease. Nature medicine. 2017;23(5):579-89. 
43. Bertani L, Fornai M, Fornili M, Antonioli L, Benvenuti L, Tapete G, et al. Serum oncostatin M at baseline predicts mucosal healing in Crohn's disease patients treated with infliximab. Alimentary pharmacology \& therapeutics. 2020;52(2):284-91.

\section{Figure Legends}

Figure 1.

Flow diagram of the study design.

Figure 2.

Nomograms for model 1 and model 2 were developed in the training cohort. (A) Nomogram 1 for model 1, with BMI, behavior, and CRP. BMI, Body Mass Index; CRP, C-reactive protein. (B) Nomogram 2 for model 2, with BMI, behavior, CRP, and IL6. IL6, interleukin-6.

Figure 3 .

ROC for model 1 and model 2 in the validation cohort. (B) Calibration plots for model 1. (C) Calibration plots for model 2. (D) DCA for model 1and model 2 in the validation cohort.

Figure 4.

GSEA analysis of $I L-6$ and $I L-6$ related genes. (B) Inflammatory response of GSEA analysis.

Figure 5 .

PPI networks for $I L-6$ and $I L-6$ related genes with the top 50 highest correlation coefficients.

Figure 6 .

Multi-factor regulation network for $I L-6$ and $O S M$.

Supplementary Figure 1.

IL6 levels were detected before and after IFX therapy in the responder and PNR groups.

Supplementary Figure 2.

DEGs in GSE111761.

Supplementary Figure 3.

GO analysis of 13 genes in the PPI directly connecting to $I L-6$. 


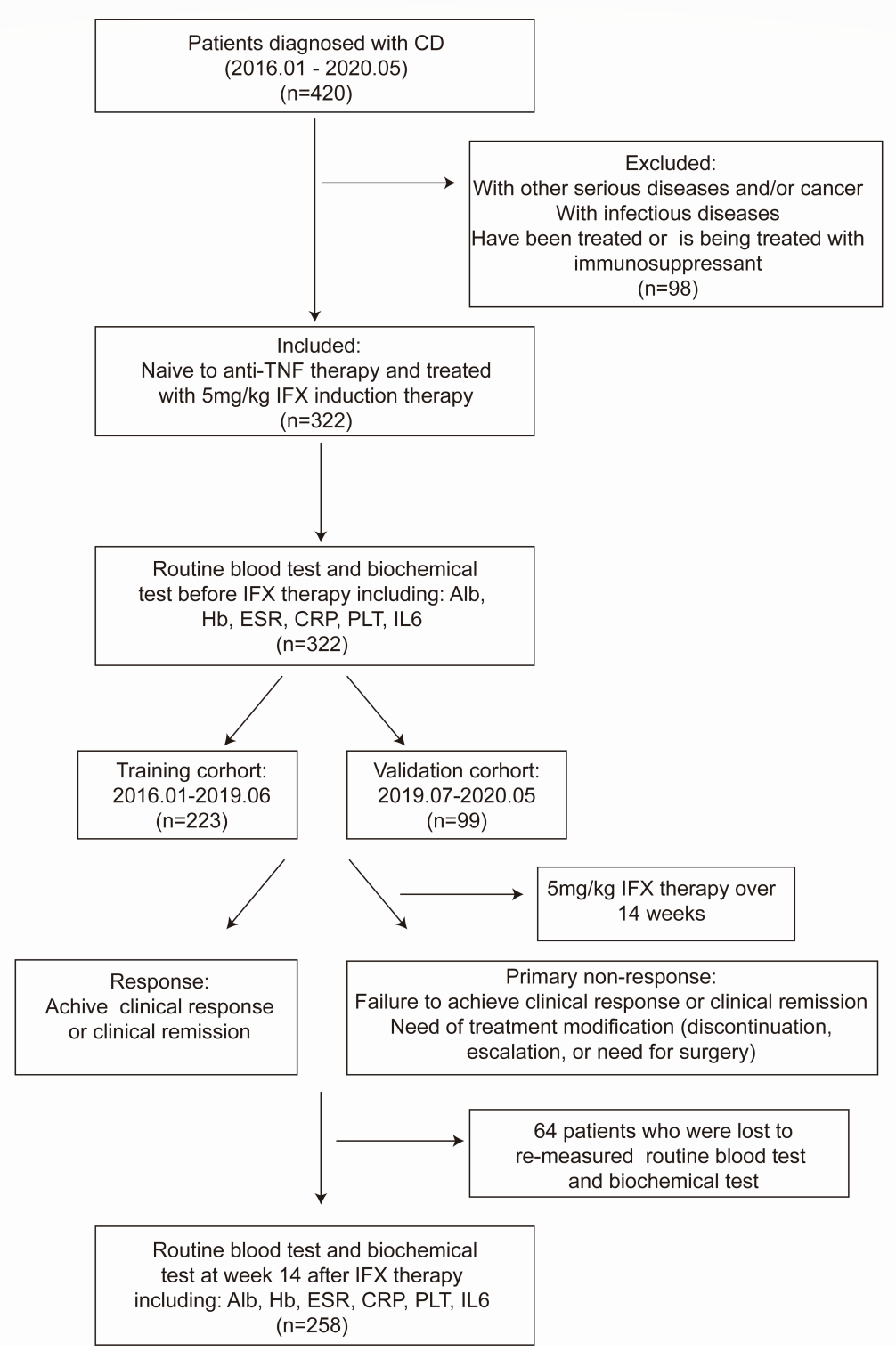




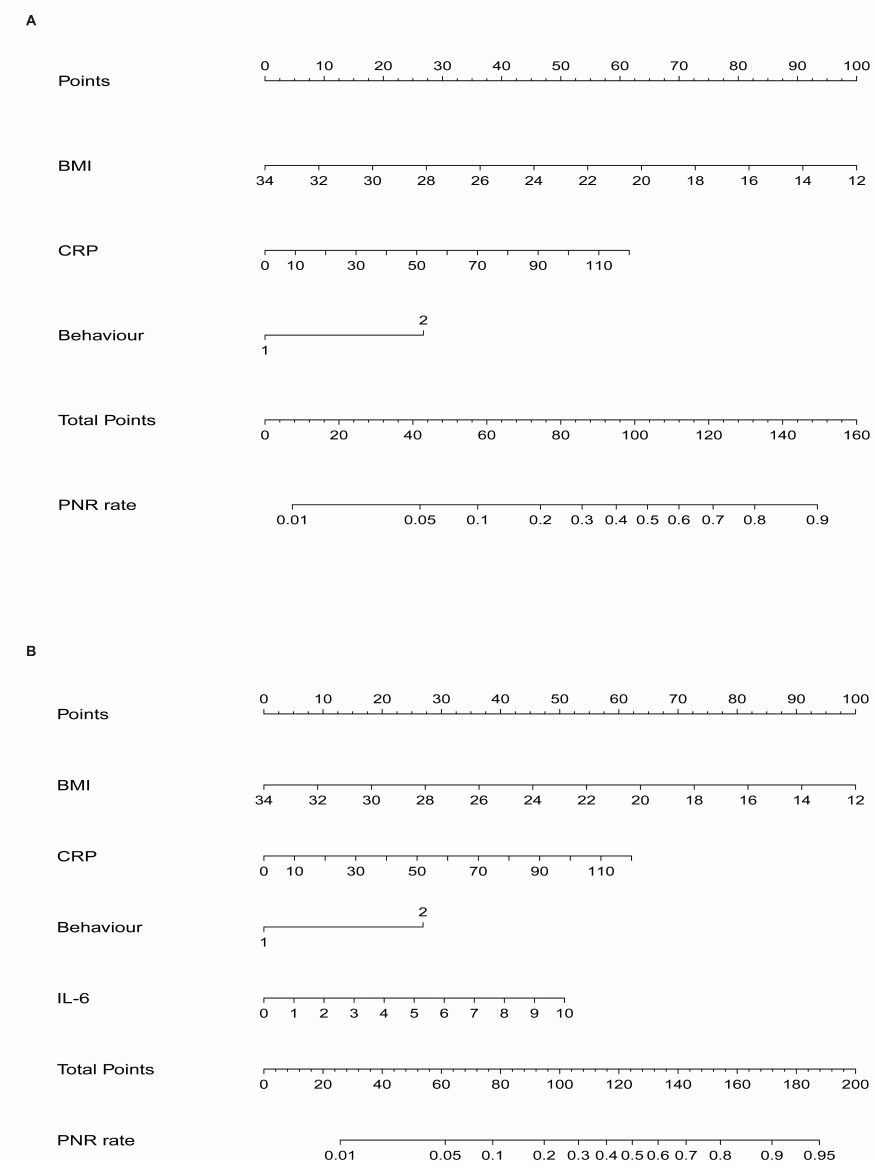



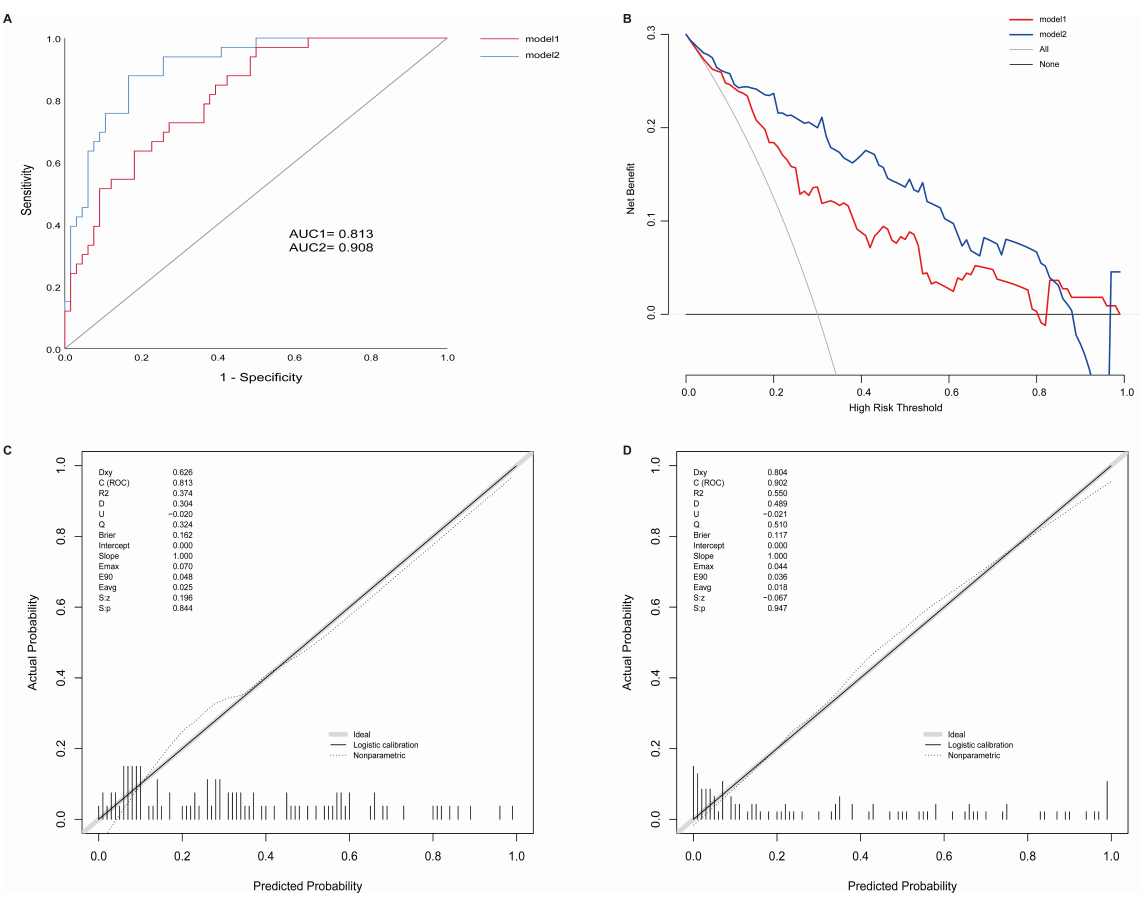
A

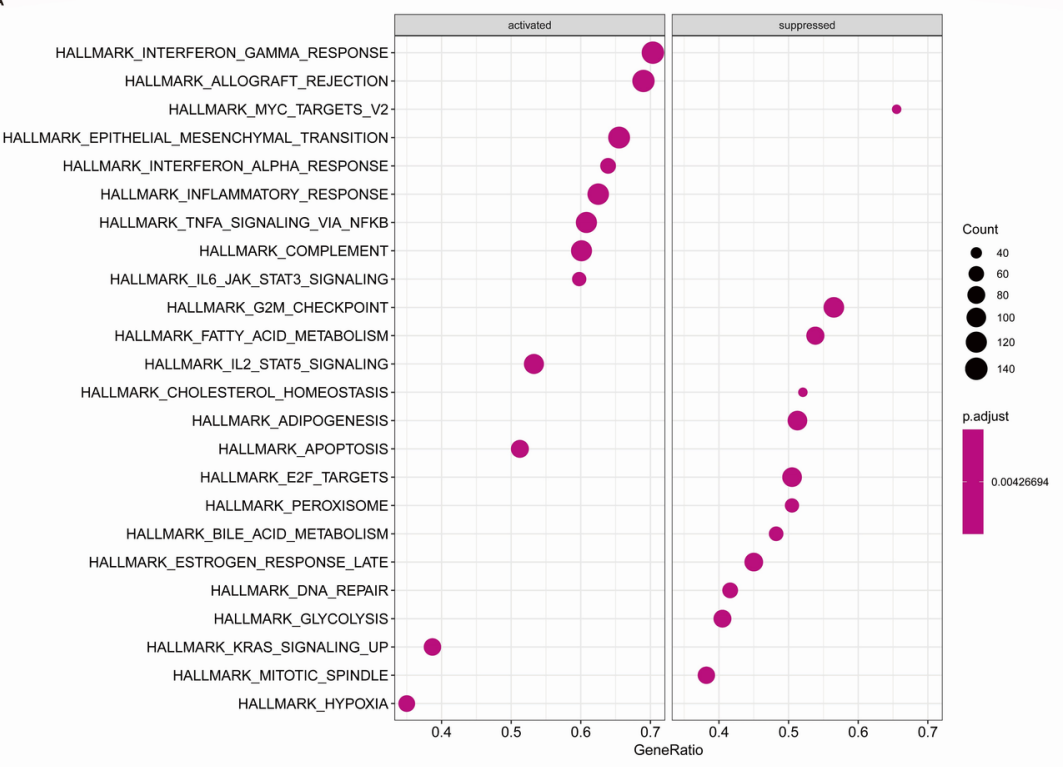

B

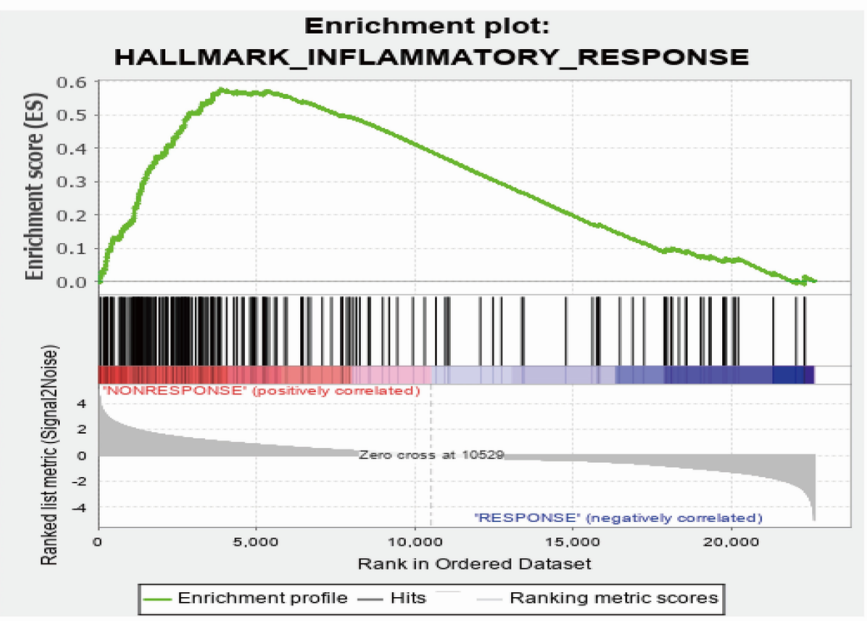



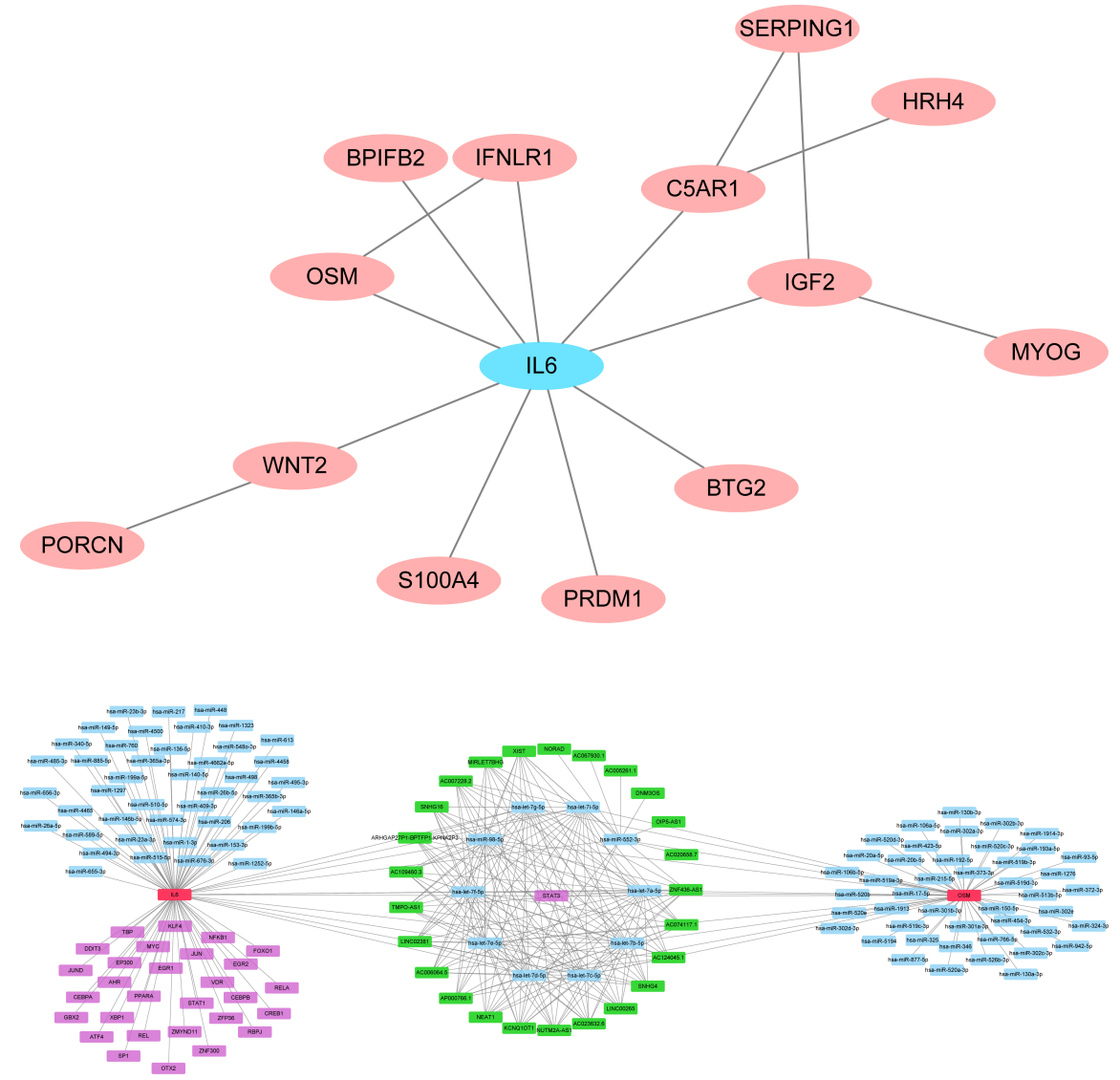

\section{Hosted file}

Table 1.pdf available at https://authorea.com/users/380269/articles/496325-development-andvalidation-of-an-interleukin-6-nomogram-to-predict-primary-non-response-to-infliximabin-crohn-s-disease-patients-from-bedside-to-bioinformatics

\section{Hosted file}

Table 2.pdf available at https://authorea.com/users/380269/articles/496325-development-andvalidation-of-an-interleukin-6-nomogram-to-predict-primary-non-response-to-infliximabin-crohn-s-disease-patients-from-bedside-to-bioinformatics

\section{Hosted file}

Table 3.pdf available at https://authorea.com/users/380269/articles/496325-development-andvalidation-of-an-interleukin-6-nomogram-to-predict-primary-non-response-to-infliximabin-crohn-s-disease-patients-from-bedside-to-bioinformatics 